\title{
Implante valvular aórtico transarterial: experiencia en Chile
}

\author{
CARLOS CAORSI ${ }^{1,2}$, CRISTIAN BAEZA ${ }^{1,2}$, CÉSAR MORÍS ${ }^{3}$, \\ MIGUEL OYONARTE ${ }^{2}$, JUAN CARLOS VENEGAS ${ }^{1}$, JORGE YOVANOVICH ${ }^{1}$, \\ JORGE RUFS ${ }^{1}$, RODRIGO DÍAZ ${ }^{1}$, MANUEL ESPÍNDOLA ${ }^{1}$
}

\section{Transcatheter aortic valve implantation. Experience in 17 patients}

Background: Transcatheter aortic valve implantation (TAVI) is taking a leading role in the management of patients with severe aortic stenosis. Valve replacement surgery prolongs survival and is the technique of choice considering its historical background and long experience worldwide. Recently however, TAVI has positioned itself as the only standard therapy for symptomatic patients who are not candidates for surgery. Aim: To report the experience with this new technique comparing our results with those reported in the literature. Material and Methods: Between May 2010 and December 2011, 17 patients aged $81 \pm 7.3$ years (58.8\% men with an Euro SCORE $29 \pm 22.4 \%$ ) underwent a TAVI. Results: The median transvalvular gradient was $54 \pm 15.7 \mathrm{mmHg}$. All patients received a CoreValve ${ }^{T M}$. Technical success was $100 \%$, with a post implant gradient of $6.29 \pm 3.3 \mathrm{mmHg}$. Residual aortic regurgitation was observed in 94\%, none greater than grade II. There were no complications at the vascular access site. One patient developed cardiac tamponade during the procedure. Permanent pacemaker implantation was required in $35.2 \%$. Hospital mortality rate was 5.8\%, a figure that remained unchanged at 30 days of follow-up. Conclusions: In high-risk patients with aortic stenosis, TAVI has a high success rate and a low rate of complications. Besides an appropriate patient selection, a trained multidisciplinary team and technical conditions to solve possible complications of the procedure are required.

(Rev Med Chile 2013; 141: 7-14).

Key words: Aortic valve replacement; Aortic valve stenosis; Balloon valvuloplasty.

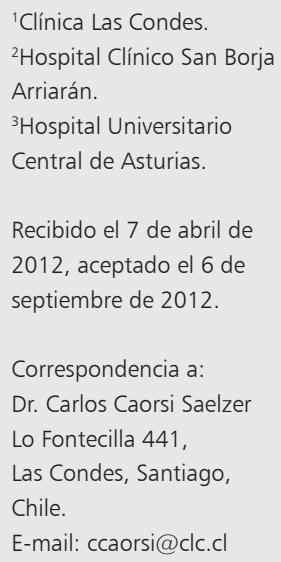

A ctualmente, la estenosis aórtica (EA) es la enfermedad valvular más frecuente en los países desarrollados, dado el aumento de las expectativas de vida. Corresponde mayoritariamente a estenosis aórtica (EA) calcificadas en pacientes mayores (2-7\% de la población mayor de 65 años $)^{1}$. El reemplazo valvular quirúrgico es aún el tratamiento de elección para pacientes con EA severa sintomática (recomendación Clase I, con nivel de evidencia B, guías ACC/AHA, Circ. 2008; 118: e523-e661).

Sin cirugía, el pronóstico es extremadamente ominoso, con una tasa de sobrevida a 3 años infe- rior a 30\%, sin embargo, 33\% de todos los pacientes mayores de 75 años con EA severa se descartan de cirugía ${ }^{2}$. La mortalidad para pacientes de alto riesgo con EA severa sintomáticos es superior a $50-60 \%$ a los 2 años. De aquellos que finalmente llegan a cirugía de reemplazo valvular, una proporción significativa tiene alta morbi-mortalidad asociada al procedimiento ${ }^{3}$.

La valvuloplastía percutánea con balón fue la primera técnica basada en catéter para resolver este problema. Luego de resultados iniciales promisorios ${ }^{4,5}$, los datos del seguimiento a largo plazo demostraron una alta tasa de re-estenosis así como 
Implante valvular aórtico transarterial - C. Caorsi et al

ausencia de mejoría clínica significativa ${ }^{6}$. Por ello, la valvuloplastía con balón es hoy considerada sólo como una opción de emergencia y como puente hacia la cirugía o el implante valvular aórtico transcatéter (TAVI). Esta nueva técnica, inicialmente descrita por Andersen ${ }^{7}$, fue introducida en el año 2008 por Cribier, en pacientes mayores y de alto riesgo quirúrgico, con estenosis aórtica severa sintomática ${ }^{8}$. Las primeras series de centros únicos demostraron la viabilidad y eficacia de la prótesis balón-expansible Sapien Edwards $s_{\mathrm{TM}}$ (Edwards Lifesciences LLC, Irvine, CA, USA) ${ }^{9}$, así como de la válvula auto-expansible CoreValve ${ }_{\mathrm{TM}}$ (Figura 1), ahora Medtronic CoreValve ${ }_{\mathrm{TM}}$ (Medtronic CoreValve, Irvine, CA, USA $)^{10-12}$. Esto fue confirmado por un gran registro multicéntrico de la CoreValve de Medtronic ${ }^{13}$. Los promisorios resultados a nivel

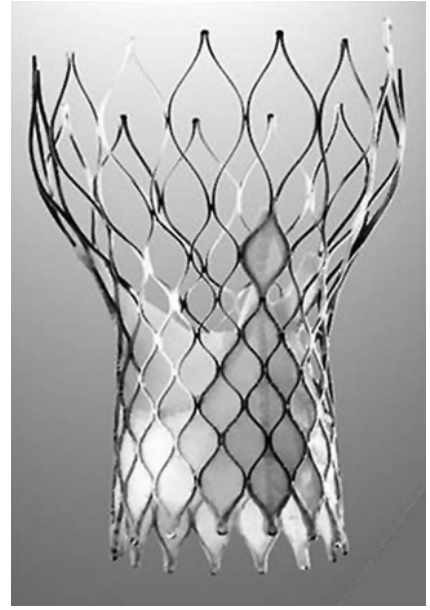

Figura 1. Prótesis Medtronic CoreValve TM. $_{\text {. }}$ mundial han determinado una rápida penetración de esta técnica en la práctica clínica habitual con un rápido crecimiento de implantes de ambas prótesis (Figura 2) ${ }^{14}$.

El objetivo del presente trabajo es presentar la experiencia inicial de un equipo cardiológicoquirúrgico en un centro único y compararla con la literatura internacional (Tabla 1).

\section{Material y Método}

\section{Población de pacientes}

Se incluyeron todos los pacientes consecutivos con estenosis aórtica severa sintomática tratados con TAVI entre mayo de 2010 y noviembre de 2011, 17,6\% referidos desde el Hospital Clínico San Borja Arriarán, 5,8\% del Hospital San José. Todos los pacientes otorgaron el consentimiento informado antes del procedimiento.

El estudio previo a la selección de los pacientes incluyó exámenes generales, ecocardiografía trans-torácica (ETT) realizados por un operador, para confirmar el diagnóstico. En la mayoría de los pacientes también se realizó una tomografía computada multi-corte (TAC). Ambos métodos, ETT y TAC, se utilizaron para evaluar las dimensiones de la aorta y de la válvula aortica, así como su morfología y el grado y distribución de las calcificaciones. Todos los pacientes fueron sometidos a coronariografía y aortografía previo al procedimiento. En más de $94 \%$ de los casos el implante valvular se realizó bajo ecocardiografía transesofágica. La decisión de tratar, así como de realizar el TAVI, se basó en el consenso del equipo.

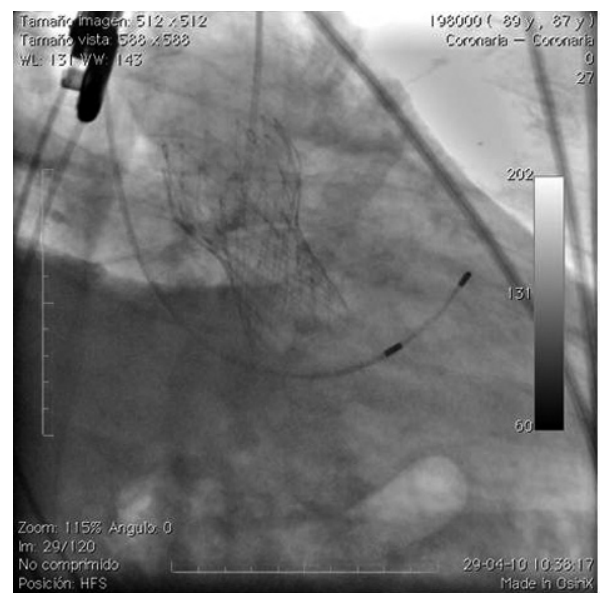

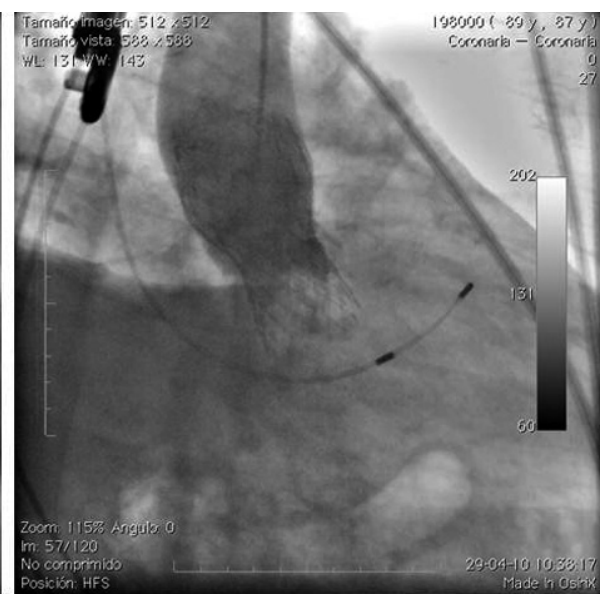

Figura 2. Primer paciente sometido a TAVI en Chile. Izquierda: imagen de valvula implantada en anillo aórtico. Derecha: aortografía demostrando cierre velos valvulares. 
Implante valvular aórtico transarterial - C. Caorsi et al

Tabla 1. Registros con sistema CoreValve (Circ J 2011; 75: 11-19)

\begin{tabular}{|c|c|c|c|c|c|c|c|c|}
\hline Registro & $\begin{array}{l}\text { Seg. } \\
\text { años }\end{array}$ & $\begin{array}{l}\text { Edad } \\
\text { (Años) }\end{array}$ & $\begin{array}{c}\text { EurosCORE } \\
\text { Logístico } \\
(\%)\end{array}$ & $\begin{array}{l}\text { LVEF } \\
(\%)\end{array}$ & $\begin{array}{c}\text { Grad. } \\
\text { media } \\
(\mathrm{mmHg})\end{array}$ & $\begin{array}{l}\text { Éxito } \\
\text { del } \\
\text { proc. } \\
(\%)\end{array}$ & $\begin{array}{l}\text { Mort. } \\
(30 \\
\text { días) } \\
(\%)\end{array}$ & MPD \\
\hline $\begin{array}{l}\text { 18F Trial Seguridad y } \\
\text { eficacia* }\end{array}$ & 2 & $81,9 \pm 6,4$ & $23,4 \pm 13,8$ & $51,6 \pm 13,1$ & $46,8 \pm 15,9$ & 83,1 & 15,2 & 31,3 \\
\hline $\begin{array}{l}\text { Estudio Australia-Nueva } \\
\text { Zelandia* }\end{array}$ & $6 \mathrm{~m}$ & $81,9 \pm 6,4$ & $18,7 \pm 12,9$ & $58,7 \pm 9,14$ & $46,4 \pm 15,9$ & 96,8 & 3,2 & 39 \\
\hline Registro italiano* & 1 & $82 \pm 6$ & $22,9 \pm 13,5$ & $51 \pm 13$ & $52 \pm 17$ & 98,1 & 7,2 & 18,5 \\
\hline Registro belga* & 1 & $82 \pm 6$ & $25 \pm 15$ & $59 \pm 13$ & $49 \pm 16$ & 98,0 & 9,0 & 26,9 \\
\hline Registro español ( $n=108)$ & 1 & $78 \pm 6,7$ & $16 \pm 13,9$ & - & $55 \pm 14,3$ & 98,1 & 7,4 & 35,2 \\
\hline Registro Frances $(n=244)$ & $6 \mathrm{~m}$ & $82,5 \pm 5,9$ & $24,7 \pm 11,2$ & $51 \pm 15$ & $46 \pm 15$ & 97,0 & 15,1 & 23 \\
\hline Registro UK* & 2 & 83 & 20,3 & - & - & 99 & 5,5 & 26 \\
\hline Registro alemán ( $n=697$ ) & $1 \mathrm{~m}$ & $81 \pm 6,4$ & $20,8 \pm 13,3$ & $52,1 \pm 15$ & $48,7 \pm 17,2$ & 98,7 & 12,4 & 42,5 \\
\hline $\begin{array}{l}\text { Hospital Universitario Cen- } \\
\text { tral de Asturias }(n=115)^{* *}\end{array}$ & $1 \mathrm{~m}$ & $82 \pm 6,3$ & $12,7 \pm 7,1$ & $59,3 \pm 11,3$ & $52,8 \pm 12,5$ & 96,8 & 6,3 & 35,5 \\
\hline $\begin{array}{l}\text { Clínica Las Condes } \\
(n=17)^{\star *}\end{array}$ & 1 & $81 \pm 7,3$ & $29 \pm 22,4$ & $53 \pm 15,2$ & $54 \pm 15,7$ & 100 & 5,8 & 35,2 \\
\hline
\end{tabular}

*presentados como abstract. ${ }^{* *}$ presentados en euroPCR-Paris 2012.

\section{Descripción del dispositivo}

La prótesis Medtronic CoreValve ${ }_{\mathrm{TM}}$ (Medtronic CoreValve) (Figura 1) es una prótesis tri-valvular biológica de pericardio porcino, montada y suturada en un marco de stent auto-expansible de nitinol. Actualmente, el sistema de liberación Acutrac (utilizado desde el segundo paciente) tiene un tamaño de 18 French. En la actualidad hay dos tamaños de dispositivos para las distintas dimensiones de anillo aórtico, una prótesis de $26 \mathrm{~mm}$ para anillos de entre 20 y $24 \mathrm{~mm}$ y otra de $29 \mathrm{~mm}$ para anillos aórticos que midan desde $24 \mathrm{a}$ $27 \mathrm{~mm}$. Recientemente se incorporó una prótesis $31 \mathrm{~mm}$ para anillos cuyo diámetro va desde 26 a $29 \mathrm{~mm}$. El procedimiento se realizó de acuerdo a la técnica y protocolo reportados ${ }^{10-12}$.

\section{Características de la población}

La edad promedio de la población fue de $81,3 \pm$ 7,3 años, $41 \%$ eran mujeres y el EuroSCORE logístico de $29,2 \%$. En cuanto a co-morbilidades, $23,5 \%$ eran diabéticos, tres $(11,7 \%)$ tenían limitación crónica del flujo aéreo y uno de ellos con oxígeno terapia permanente. Tres pacientes $(17,6 \%)$ tenían insuficiencia renal crónica, accidente vascular previo o enfermedad vascular periférica obstructiva.
Cuatro de los pacientes habían tenido cirugía de revascularización miocárdica y uno reemplazo valvular aórtico. Tres habían sido anteriormente sometidos a angioplastía coronaria y uno a valvuloplastía aórtica con balón. Insuficiencia cardiaca Clase $\geq$ III de la New York Heart Association (NYHA), estaba presente en $88 \%$ de los pacientes. Las principales indicaciones de TAVI fueron alto riesgo quirúrgico, 41,1\% EuroSCORE 20-30\%, y contraindicación de cirugía en 41,1\% EuroSCORE $>30$ (Tabla 2). Todos los pacientes fueron analizados por un "heart team"; se consideró que siete de ellos tenían contraindicación quirúrgica por EuroSCORE, edad y comorbilidad, el promedio de EuroSCORE de estos pacientes fue de 47,3\% (rango 33-83). Tres pacientes rechazaron la cirugía convencional, EuroSCORE $\mathfrak{E} 20 \%$, corresponden a NYHA II.

La fracción de eyección promedio fue de $53 \pm$ $15,1 \%$, sin embargo, 3 (17,6\%) de los pacientes presentaban una función ventricular severamente deprimida con valores menores a 30\%. La gradiente trans-valvular máxima promedio fue de 79,3 \pm $20 \mathrm{mmHg}$ y la media de $54 \pm 15,7$, con velocidades máximas promedio de $4,1 \pm 1,0 \mathrm{~m} / \mathrm{s}$. El promedio del diámetro del anillo fue de 24,8 $\pm 2,2 \mathrm{~mm}$ (rango 
Tabla 2. Características de los pacientes

\begin{tabular}{|lc|}
\hline & (n = 17) \\
Edad (años) & $81,3 \pm 7,3$ \\
Sexo masculino (\%) & 58,8 \\
IMC (kg/m²) & $25,5 \pm 4,3$ \\
Historia médica & \\
Cirugía bypass previa (\%) & $4(23,5)$ \\
Angioplastia previa (\%) & $3(17,6)$ \\
Cirugía valvular previa (\%) & $1(5,8)$ \\
Valvuloplastía con balón previa (\%) & $1(5,8)$ \\
Accidente vascular previo (\%) & $3(17,6)$ \\
Diabetes mellitus (\%) & $4(23,5)$ \\
LCFA (\%) & $2(11,7)$ \\
Enfermedad vascular periférica obstructiva (\%) & $3(17,6)$ \\
Insuficiencia renal (\%) & $3(17,6)$ \\
Marcapasos previo (\%) & $2(11,7)$ \\
Presentación Clínica & \\
Angina (\%) & $7(41,1)$ \\
Sincope (\%) & $3(17,6)$ \\
NYHA II (\%) & $2(11,7)$ \\
NYHA III (\%) & $9(52,9)$ \\
NYHA IV (\%) & $6(35,3)$ \\
EuroSCORE Logístico (\%) & $7(41,1)$ \\
Indicación de TAVI (\%) & 29,2 \\
Riesgo quirúrgico alto (EuroSCORE 20\%) (\%) & $7(41,1)$ \\
Decisión paciente (\%) & $3(17,6)$ \\
Contraindicación para cirugía (\%) & 7 \\
\hline & \\
\hline
\end{tabular}

Tabla 3. Hallazgos hemodinámicos y angiográficos

\begin{tabular}{|lc|}
\hline & (n = 17) \\
\hline FEVI & \\
Fracción de eyección (\%) & $53 \pm 15,1$ \\
Reducción severa (<30\%) & $3(17,6)$ \\
Características de la válvula & \\
Área valvular cm ${ }^{2}$ & \\
Gradiente máx. (mmHg) & $0,58 \pm 0,25$ \\
Gradiente medio (mmHg) & $79,3 \pm 20$ \\
Válvula bicúspide (\%) & $54 \pm 15,7$ \\
Calcificación severa (\%) & $1 \quad(5,8)$ \\
Diámetro anillo (mm) & $13(76,4)$ \\
Bajo debito-estenosis de gradiente bajo (\%) & $24,8 \pm 2,2$ \\
Insuficiencia aórtica concomitante grado $\geq 3(\%)$ & $4(23,5)$ \\
Otros hallazgos & $1(5,8)$ \\
Insuficiencia mitral concomitante Grado $\geq 3(\%)$ & $3(17,6)$ \\
Presión sistólica arteria pulmonar (mmHg) & $47,8 \pm 14,5$ \\
Hipertensión pulmonar (> 50 mmHg) & $6(35,3)$ \\
Fibrilación auricular (\%) & $4(23,5)$ \\
Marcapaso previo (\%) & $2(11,7)$ \\
\hline
\end{tabular}

22-29 mm) (Tabla 3). Un paciente era portador de una válvula aortica bicúspide, y 53\% de los pacientes presentaba en forma concomitante insuficiencia valvular aórtica grado I a II. Insuficiencia mitral mayor a grado II se observó en $58,8 \%$. La presión sistólica de arteria pulmonar (PSAP) promedio fue de 47,88 $\pm 14,5 \mathrm{mmHg}$. El 35,3\% de los pacientes tenían una PSAP $\geq 50$ $\mathrm{mmHg}$ y dos de ellos $>$ de $70 \mathrm{mmHg}$. Dos pacientes tenían marcapasos cardiaco definitivo y el procedimiento y cuatro se encontraban en fibrilación auricular previo al procedimiento.

\section{Resultados}

En el 100\% de los casos el acceso vascular fue abordado por acceso femoral mínimo, teniendo en consideración que la principal complicación de esta técnica se presenta en el sitio de acceso (11\%) La suma de las complicaciones vasculares y hemorrágicas de este procedimiento, dan cuenta del aproximadamente el 20\% de las complicaciones de la técnica.

\section{Datos del procedimiento}

Todos los pacientes fueron abordados por acceso femoral quirúrgico mínimo, a pesar de la patología vascular periférica este abordaje fue posible en todos los pacientes. No se observaron complicaciones vasculares locales ni circulatorias distales durante el procedimiento o en la evolución posterior.

El implante valvular fue principalmente un procedimiento electivo $(94,2 \%)$, sólo en un paciente $(5,8 \%)$ se realizó de emergencia. Este paciente se encontraba en edema pulmonar y tenía una válvula aórtica Mitroflow \#21 disfuncionante. $\mathrm{Al}$ momento de realizarlo existía sólo un caso más reportado en la literatura, por lo que se contactó al autor y a otros expertos internacionales. Este caso en particular se realizó sin balonplastía previa y se implantó directamente. El tiempo medio de duración de la intervención fue de 126,8 \pm 30,2 min. El tiempo desde la instalación del introductor, hasta el implante de la válvula fue de 61,8 \pm 20,2 min, con un tiempo medio de radioscopia de $24,4 \pm 5,7 \mathrm{~min}$. Se utilizó en promedio 214 $\pm 88 \mathrm{ml}$ de medio de contraste, Visipaque ${ }^{\circledR}$. El éxito técnico, definido como el implante de 
la válvula se logró en $100 \%$. La gradiente trans-aórtica residual post procedimiento fue de 6,3 $\pm 3,4 \mathrm{mmHg}$. El implante directo, sin balonplastía previa, se utilizó en $11,7 \%$ de los casos. En 35,3\% se utilizó una CoreValve \#26 y en $64,7 \%$ una CoreValve \#29. La insuficiencia aortica residual al final del procedimiento se observó en $94 \%$ de los procedimientos, sin embargo, no se observó insuficiencia aórtica severa ( $\geq$ grado III) (Tabla 4 ).

Tabla 4. Procedimiento y eventos clínicos

\begin{tabular}{|c|c|}
\hline & $(n=17)$ \\
\hline Electiva (\%) & $16(94,1)$ \\
\hline Urgente (\%) & $1 \quad(5,88)$ \\
\hline \multicolumn{2}{|l|}{ Tipo de válvula } \\
\hline CoreValve 26 (\%) & $5(35,3)$ \\
\hline CoreValve $29(\%)$ & $11(64,7)$ \\
\hline Valvuloplastía previa (\%) & $15(88,3)$ \\
\hline Implantación directa (\%) & $2(11,7)$ \\
\hline \multicolumn{2}{|l|}{ Características procedimiento } \\
\hline Tiempo intervención (min.) & $126,8 \pm 30,2$ \\
\hline Tiempo de fluoroscopio (min.) & $24,4 \pm 5,7$ \\
\hline Medio contraste $(\mathrm{ml})$ & $214 \pm 88$ \\
\hline Cierre quirúrgico sitio punción (\%) & $17(100)$ \\
\hline \multicolumn{2}{|l|}{ Resultados procedimiento } \\
\hline Éxito procedimiento (\%) & $17(100)$ \\
\hline Gradiente post procedimiento (\%) & $6,29 \pm 3,3$ \\
\hline Insuficiencia residual aortica (\%) & $16(94)$ \\
\hline Grado I (\%) & $14(82,3)$ \\
\hline Grado II (\%) & $3(17,6)$ \\
\hline Implante marcapasos (\%) & $5(35,3)$ \\
\hline \multicolumn{2}{|l|}{ Curso clínico } \\
\hline Tiempo en UCC (días) & $2,25 \pm 0,94$ \\
\hline Necesidad de transfusión (\%) & 0 \\
\hline Necesidad soporte hemodinámico IABP ECMO (\%) & 0 \\
\hline Taponamiento pericárdico (\%) & $1 \quad(5,88)$ \\
\hline Disección aortica (\%) & 0 \\
\hline Isquemia coronaria (\%) & 0 \\
\hline Ruptura anillo (\%) & 0 \\
\hline Embolización (\%) & 0 \\
\hline Insuficiencia renal (\%) & $1 \quad(5,88)$ \\
\hline Accidente cerebrovascular (\%) & $1 \quad(5,88)$ \\
\hline Trombo embolismo pulmonar (\%) & 0 \\
\hline Muerte intrahospitalaria (\%) & $1 \quad(5,88)$ \\
\hline Muerte a 30 días (\%) & $1 \quad(5,88)$ \\
\hline Muerte seguimiento (\%) & $1 \quad(5,88)$ \\
\hline
\end{tabular}

\section{Complicaciones y evolución clínica}

El tiempo medio de estadía en la Unidad Coronaria fue de $2,25 \pm 0,94$ días y el de hospitalización de $6 \pm 2,79$ días. La mayoría de los pacientes recibió terapia anti-agregante dual (aspirina y clopidogrel). Se implantó marcapasos definitivo en $35,2 \%$ de los pacientes. No se observaron disecciones aórticas y un paciente presentó taponamiento pericárdico, el que se resolvió instalando un drenaje. (Se han reportado tasas de taponamiento de $1,8 \%-2,0 \%)^{15}$. En general, todos los pacientes mejoraron su capacidad funcional (CF) en dos grados. Como se describe para este procedimiento, la mejoría de la capacidad funcional se presenta precozmente post implante siendo notorio ya a partir del primer mes. El seguimiento clínico se cumplió en $94 \%$ a los 6 meses, $82 \%$ a un año. Un paciente no mejoró su capacidad funcional manteniendo una insuficiencia mitral severa y mala fracción de eyección. En este caso los estudios iniciales de insuficiencia aortica residual fueron contradictorios, como se describe también en otras publicaciones, y posteriormente se demostró una insuficiencia aórtica mayor a grado II, por lo que se implanto válvula en válvula en otro centro, debiendo también efectuarse re-sincronización ventricular para mejorar su función. No se registraron accidentes vasculares clínicos. La mortalidad intra-hospitalaria fue de 5,88\%, dada por un paciente que evolucionó con deterioro de la función renal y en quien la familia decidió no efectuar diálisis. La mortalidad a 30 días fue de 5,88\%, en relación a este caso y la muerte a un año de seguimiento fue de $11,7 \%$ por haberse sumado un paciente que falleció de insuficiencia cardiaca a los 6 meses (Tabla 4).

\section{Discusión}

Actualmente, los registros son un método aceptado y recomendado para monitorizar y obtener importante información del desempeño de nuevas terapias médicas, en pacientes no seleccionados del "mundo real”, en centros no-especializados ${ }^{15}$. En 
nuestra serie, la edad media, es similar a lo comunicado en otros registros (Tabla 1), sin embargo, el EuroSCORE es mayor considerando la avanzada edad de algunos pacientes y sus co-morbilidades. La rigurosa selección de los pacientes determinó que $82,3 \%$ de ellos tenía un riesgo quirúrgico elevado o contraindicación quirúrgica. Piazza et $\mathrm{al}^{16}$ reportaron el uso de TAVI en $67 \%$ de casos "off-label", vale decir sin indicación de consenso. Se ha reportado que hasta $13 \%$ de los pacientes han decidido realizarse un TAVI a pesar de haber sido casos adecuados para un manejo quirúrgico. En nuestra serie tres pacientes rechazaron la cirugía, destacando el caso de una paciente de 62 años que rechazo la cirugía y optó por TAVI por razones religiosas, caso que debió ser presentado al Comité de Ética. Sin embargo, en este momento las indicaciones "off-label", en los centros de bajo volumen y que están comenzando su experiencia debieran ser evitadas.

En relación al estudio previo al procedimiento, en nuestra experiencia la tomografía multicorte (TAC) aportó datos sobre las dimensiones y calcificación de los accesos vasculares, así como también sobre la distribución del calcio valvular y morfología de la aorta ascendente; en cambio el aporte de la TAC en la decisión del tamaño de la válvula seleccionada fue menor, dado que el anillo es ovoideo. Nuestro grupo se basó fundamentalmente en la medición de la ecografía transesofágica intra procedimiento para decidir el tamaño de la válvula.

La lista de posibles complicaciones graves en TAVI es extensa: embolia cerebral, taponamiento pericárdico, insuficiencia aórtica severa, disección aórtica, ruptura cardiaca, así como el acceso vas-

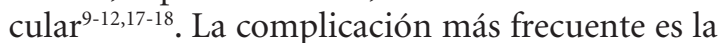
necesidad de implantar un marcapasos definitivo por el bloqueo $\mathrm{AV}$ de tercer grado intermitente o persistente. Cuando se utilizó CoreValve llegó a hasta $42 \%{ }^{15}$. En nuestro grupo se implantó marcapasos definitivo en $35,2 \%$ de los pacientes, lo que está dentro de los rangos reportados por otros centros. En nuestra serie, dos pacientes tenían marcapasos previo al procedimiento, un paciente presentó bloqueo antes de TAVI y cuatro tenían una fibrilación auricular previa. La mayor necesidad de implante de marcapasos con el dispositivo CoreValve puede estar relacionada a mayores y más perdurables fuerzas radiales, así como a la implantación más profunda hacia el tracto de salida del ventrículo izquierdo, de la válvula auto-expandible Medtronic CoreValve ${ }_{\mathrm{TM}}$, lo cual puede interrumpir más frecuentemente la conducción de las ramas del haz de His. En nuestra experiencia en muchas ocasiones preferimos instalar MPD ante bloqueos transitorios post-implante para evitar mortalidad luego de un procedimiento endovascular exitoso.

Una de las principales complicaciones de TAVI es la insuficiencia aórtica, la cual se observa hasta en $72,4 \%$ de los pacientes ${ }^{15}$. En nuestra serie se observó 94\% de insuficiencia aórtica grado I o II, sin repercusiones clínicas en el seguimiento. En un caso la insuficiencia fue mayor por lo que se debió reposicionar y traccionar la válvula con la técnica del "lazo" con lo que se logró corregir. La evaluación de la insuficiencia residual se basó en ecografía transesofágica y aortografía. Siempre se consideró durante el procedimiento que la insuficiencia residual era relevante ( $>$ grado II) se postdilató con balón hasta lograr un resultado adecuado. El diagnóstico exacto de la severidad de esta insuficiencia, así como su tratamiento, son dos de los problemas más desafiantes de TAVI. Los datos disponibles de ambos tipos de válvulas son similares en este sentido, alcanzando tasas aproximadas de 70\%, con insuficiencia leve a moderada ${ }^{9,12,20}$. La insuficiencia aórtica severa se ha reportado en tasas de $2,3 \%{ }^{15}$, sin embargo, este valor puede subestimar el verdadero problema por dos razones: primera, la insuficiencia severa que es tratada durante la implantación no se reporta y segunda insuficiencias inicialmente estimadas como moderadas en algunos casos pueden hacerse severas durante el seguimiento. La insuficiencia aórtica severa por implantación profunda de la válvula Medtronic CoreValve ${ }_{\mathrm{TM}}$ puede ser tratada tanto con la implantación de una segunda válvu$\mathrm{la}^{12,21}$ o por reposicionamiento mediante un caté$\operatorname{ter}^{11,13}$. Las fugas (leaks) para-valvulares pueden ser tratadas con un sistema de cierre percutaneo ${ }^{22,23}$. Actualmente, se considera que no es óptimo dejar una válvula post implante con insuficiencia mayor a grado II. No sólo influye la insuficiencia sino la capacidad del paciente de adaptarse a ésta en la fase aguda, ya que un paciente sin insuficiencia aórtica previa tiene menor tolerancia a ello que uno con doble lesión valvular aórtica previa. El registro alemán demostró que la insuficiencia aórtica residual (definida como moderada o severa) es un predictor independiente de mortalidad intra-hospitalaria ${ }^{24}$. Insuficiencias aórticas (IA) 
leves post TAVI parecen tener efectos menores, en cambio las moderadas y severas inducen un cambio al ventrículo izquierdo de sobrecarga de presión a sobrecarga de volumen. La prevalencia de IA moderada a severa post TAVI va desde 6 a $21 \%$. Recientemente se ha destacado el impacto de estas insuficiencias moderadas a severas en la sobrevida y pobre respuesta al tratamiento ${ }^{25}$. Aún falta información sobre la evolución de los pacientes con insuficiencia aórtica residual leve, en el largo plazo. En nuestra serie, todos los pacientes, salvo uno, tuvieron insuficiencia residual inferior o igual a grado II (Tabla 4). La mortalidad hospitalaria reportada va desde $8,2 \%$ a $12,7 \%(15,26)$ siendo de $8 \%$ a $12,4 \%$ a 30 días ${ }^{13,15}$, algo superior a la nuestra de $5,88 \%$. Los predictores independientes de mortalidad a un año son: acceso distinto al femoral, fracción de eyección baja, enfermedad bronquial obstructiva crónica, insuficiencia aórtica moderada a severa postprocedimiento ${ }^{25}$. En general, la mortalidad pareciera más asociada a la avanzada edad y severas co-morbilidades de los pacientes y en menor medida a las complicaciones de la intervención misma ${ }^{15}$. Las cifras de nuestra serie son concordantes con esta hipótesis dado que los procedimientos fueron exitosos y sin complicaciones inmediatas relevantes. Esto refuerza la necesidad de una adecuada selección de los pacientes, del cuidado del paciente después de la intervención ${ }^{27}$, y de contar con personal técnicamente preparado y una adecuada infraestructura para tratar las posibles complicaciones.

Los extraordinarios resultados de TAVI explicarían la velocidad, sin precedentes, con que la técnica se ha ido incorporando a la práctica clínica. El crecimiento exponencial de las TAVI a nivel mundial desde aproximadamente 10.000 el 2010 a más de 80.000 válvulas implantadas hasta ahora en la población de mayor riesgo, sólo demuestra su eficiencia y seguridad, por lo que probablemente estemos asistiendo a un cambio conceptual en el enfoque de esta patología.

\section{Referencias}

1. Vahanian A, Baumgartner H, Bax J, Butchart E, Dion R, Filippatos G, et al. Guidelines on the management of valvular heart disease The Task Force on the Management of Valvular Heart Disease of the European Society of Cardiology. EurHeart J 2007; 28: 230-68.
2. Iung B, Baron G, Butchart EG, Delahaye F, GohlkeBarwolf C, Levang OW, et al. A prospective survey of patients with valvular heart disease in Europe: The Euro Heart Survey on valvular heart disease. Eur Heart J 2003; 24: 1231-43.

3. Varadarajan P, Kapoor N, Bansal RC, Pai RG. Survival in elderly patients with severe aortic stenosis is dramatically improved by aortic valve replacement: Results from a cohort of 277 patients aged $\geq 80$ years. Euro J CardiothoracSurg2006; 30: 722-7.

4. Cribier A, Savin T, Saoudi N, Rocha P, Berland J, Letac $B$. Percutaneous transluminal valvuloplasty of acquired aortic stenosis in elderly patients: an alternative to valve replacement? Lancet 1986; 1: 63-7.

5. NHLBI Balloon Valvuloplasty Registry Investigators Participants. Percutaneous balloon aortic valvuloplasty. Acute and 30-day follow-up results in 674 patients from the NHLBI Balloon Valvuloplasty Registry. Circulation 1991; 84: 2383-97.

6. Otto CM, Mickel MC, Kennedy JW, Alderman EL, Bashore TM, Block PC, et al. Three-year outcome after balloon aortic valvuloplasty. Insights into prognosis of valvular aortic stenosis. Circulation 1994; 89: 642-50.

7. Andersen HR, Knudsen LL, Hasenkam JM. Transluminal implantation of artificial heart valves. Description of a new expandable aortic valve and initial results with implantation by catheter technique in closed chest pigs. Eur Heart J 1992; 13: 704-8.

8. Cribier A, Eltchaninoff H, Bash A, Borenstein N, Tron C, Bauer F, et al. Percutaneous transcatheter implantation of an aortic valve prosthesis for calcific aortic stenosis: first human case description. Circulation 2002; 106: 3006-8.

9. Webb JG, Pasupati S, Humphries K, Thompson C, Altwegg L, Moss R, et al. Percutaneous transarterial aortic valve replacement in selected high-risk patients with aortic stenosis. Circulation 2007; 116: 755-63.

10. Grube E, Laborde JC, Gerckens U, Felderhoff T, Sauren B, Buellesfeld L, et al. Percutaneous implantation of the CoreValve self-expanding valve prosthesis in high-risk patients with aortic valve disease: the Siegburg first-inman study. Circulation 2006; 114: 1616-24.

11. Grube E, Schuler G, Buellesfeld L, Gerckens U, Linke A, Wenaweser P, et al. Percutaneous aortic valve replacement for severe aortic stenosis in high-risk patients using the second-and current third-generation selfexpanding CoreValve prosthesis: device success and 30day clinical outcome. J Am Coll Cardiol 2007; 50: 69-76.

12. Grube E, Buellesfeld L, Mueller R, Sauren B, Zickmann B, Nair D, et al. Progress and current status of percutaneous aortic valve replacement: results of three device 
Implante valvular aórtico transarterial - C. Caorsi et al

generations of the CoreValve Revalving system. Circ Cardiovasc Interv 2008; 1: 167-75.

13. Piazza N, Grube E, Gerckens U, den Heyer P, Linke A, Luha $\mathrm{O}$, et al. Prodedural and 30-day outcomes following transcatheter aortic valve implantation using the third generation (18F) CoreValve Revalving system: results from the multicentre, expanded evaluation registry 1-year following CE mark approval. EuroIntervention 2008; 4: 242-9.

14. Serruys PW, Piazza N, Cribier A, Webb JG, Laborde JC, de Jaegere P, et al (eds). Transcatheter Aortic Valve Implantation. Tips and Tricks to Avoid Failure. New York: Informa Healthcare; 2010.

15. Zahn R, Gerckens U, Grube E, Linke A, Sievert H, Eggebrecht $\mathrm{H}$, et al. Transcatheter aortic valve implantation: first results from a multi-centre real-world registry. Eur H J 2011; 32: 198-204.

16. Piazza N, Otten A, Schultz C, Onuma Y, García GH, Boersma E, et al. Adherence to patient selection criteria in patients undergoing transcatheter aortic valve implantation with the $18 \mathrm{~F}$ CoreValve RevalvingTM System-Results from a single-center study. Heart 2010; 96: 19-26.

17. Masson JB, Kovac J, Schuler G, Ye J, Cheung A, Kapadia $S$, et al. Transcatheter aortic valve implantation: review of the nature, management, and avoidance of procedural complications. JACC Cardiovasc Interv 2009; 2: 811-20.

18. Zahn R, Schiele R, Kilkowski C, Zeymer U. Aortic insufficiency after transcatheter aortic valve implantation: on the importance to clarify the underlying pathophysiology. Clin Res Cardiol 2010; 99: 193-7.

19. Kahlert P, Knipp SC, Schlamann M, Thielmann M, AlRashid F, Weber M, et al. Silent and apparent cerebral ischemia after percutaneous transfemoral aortic valve implantation: a diffusion-weighted magnetic resonance imaging study. Circulation 2010; 121: 870-878.

20. Webb JG, Altwegg L, Boone RH, Cheung A, Ye J, Lichtenstein S, et al. Transcatheter aortic valve implantation: impact on clinical and valve-related outcomes. Circulation 2009; 119: 3009-16.

21. Piazza N, Schultz C, de Jaegere PP, Serruys PW. Implantation of two selfexpanding aortic bioprosthetic valves during the same procedure-Insights into valve-in-valve implantation ("Russian doll concept"). Catheter Cardiovasc Interv 2009; 73: 530-9.

22. Shapira Y, Hirsch R, Kornowski R, Hasdai D, Assali A, Vaturi $\mathrm{M}$, et al. Percutaneous closure of perivalvular leaks with Amplatzer occluders: feasibility, safety, and short-term results. J Heart Valve Dis 2007; 16: 305-13.

23. Hammerstingl C, Werner N, Nickenig G. Symptomatic paravalvular leakage after mechanical aortic valve replacement in a critically ill patient: why not just "plug" the hole? Eur J Echocardiogr 2009; 10: 576-8.

24. Abdel-Wahab M, Zahn R, Horack M, et al, for the German Transcatheter Aortic Valve Interventions Registry Investigators. Aortic regurgitation after transcatheter aortic valve implantation: incidente and early outcome. Results from the German transcatheter aortic valve interventions registry. Heart 2011; 97: 899-906.

25. Gotzmann M, Lindstaedt M, Mügge A. From pressure overload to volume overload: Aortic regurgitation after transcatheter aortic valve implantation. Am Heart J 2012; 163: 903-11.

26. Eltchaninoff $\mathrm{H}$. on behalf of the FRANCE Registry Investigators. FRANCE Registry: trans-catheter aortic valve implantation in France. Early results. [Abstract]. Circulation 2009

27. Webb J, Cribier A. Percutaneous transarterial aortic valve implantation: what do we know? Eur H J 2011; 32: 1407. 